\title{
Per una grammatica lessicalmente esaustiva sull'inganno e la menzogna in italiano
}

\section{Emilio D’Agostino}

Università degli Studi di Salerno

\section{Abstract}

Nel corso della propria vita, ognuno ha a che fare con emozioni, sensazioni, comportamenti e, quindi, più in particolare, con le parole che lo mettono di fronte a ciò che tanto la propria cultura, quanto le rappresentazioni che da questa discendono classificano come menzogna, verità, falsità, passione, ira, desiderio, inganno, bugia, sdegno, impulso, sentimento, emozione, odio ecc. A queste parole del vocabolario comune corrispondono, in realtà, dei costrutti mentali che ci appaiono come dei grandi apparati significanti. Per questo contributo, si è scelto un tema tipico del discorso filosofico, letterario, psicologico, antropologico, semiologico e sociologico: la menzogna. In realtà, la linguistica si colloca, in un certo senso, in una dimensione che costituisce un osservatorio particolare e privilegiato cui non è possibile rinunciare. Essa, infatti, si trova in una condizione specialmente favorita, poiché possiede la strumentazione necessaria per descrivere minuziosamente quanto una lingua mette a disposizione dei parlanti quando questi, per i casi delle loro vite, s'imbattono nelle nebbie della bugia, dell'ira, della passione amorosa, di quella politica ecc. Cioè quanto una lingua rende disponibile all'uso comune.

Parole chiave: vocabolario, menzogna, cor duplex, operatori, lessico grammatica.

\section{Abstract}

Over the course of life, each and every one of us has a certain relation with emotions, sensations, behaviour and thus, in particular, with the words that each of us place in front of that which both in the culture itself, as well as in the representations that come from these, are classified as lies, truth, falsity, passion, ire, desire, trickery, untruths, indignation, impulse, sentiment, emotion, hatred etc. These items of common vocabulary in reality correspond to mental constructs that appear as the greater apparatus of meaning. This current article has chosen a subject typical to the discourses of philosophy, literature, psychology, anthropology, semiotics and sociology: the lie. In fact, linguistics is situated, in a certain sense, within a dimension that constitutes a most particular and privileged observatory, an opportunity that should not be missed. Linguistics actually finds itself in an especially favoured condition, as it possesses the instrumentation required to minutely describe that which a language places at the disposal of its speakers when, in the normal course of their daily lives, they find themselves caught up in the mists of untruth, anger, amorous or political passion, etc. That is, it can describe everything that a language makes available to common use.

Key words: vocabulary, lies, cor duplex, operators, grammatical lexis. 
a Maurice Gross

\subsection{Verità, passioni, inganni}

Nel corso della propria vita, ognuno ha a che fare con emozioni, sensazioni, comportamenti e, quindi, più in particolare, con le parole che lo mettono di fronte a ciò che tanto la propria cultura, quanto le rappresentazioni che da questa discendono classificano come menzogna, verità, falsità, passione, ira, desiderio, inganno, bugia, sdegno, impulso, sentimento, emozione, odio ecc. A queste parole del vocabolario comune corrispondono, in realtà, dei costrutti mentali che ci appaiono come dei grandi apparati significanti.

Per questo contributo, la ricerca su verità, passioni e inganni cui qui faccio riferimento ${ }^{1}$ prende lo spunto, come accade in molti casi, dalle suggestioni sorte a seguito di letture che hanno la capacità di sollecitare in modo significativo la riflessione e di farne, quindi, discendere altre a cascata. In particolare, in questo caso, penso a due volumi che solo in apparenza non hanno nulla a che vedere fra loro: il primo, Storia delle passioni, curato da Silvia Vegetti Finzi, ${ }^{2}$ il secondo, Filosofia della bugia, di Andrea Tagliapietra. ${ }^{3}$ In un caso come nell'altro, gli oggetti teorici analizzati sembrano riguardare delle nebulose estranee agli oggetti tipicamente analizzati dalla scienza: la Passione, intesa qui come iperonimo di una classe di sentimenti in qualche modo estremi, che viene comunemente contrapposta, anche dal senso comune, alla Ragione e, dall'altro lato, l'inganno e la menzogna che comunemente sono messi in contrapposizione alla Verità.

A ben guardare, c'è, però, come ponte tra i due oggetti una categoria che, in un senso particolare, li unisce e li rende potenzialmente membri della stessa classe, per l'appunto la categoria della Verità. Se il mentire, l'ingannare, l'occultare sono apparsi comunemente soltanto come semplici negazioni o manipolazioni interessate della Verità, allo stesso modo, la Ragione soltanto è stata ritenuta portatrice di Verità, mentre le varie passioni di cui l'uomo è in balía, invece, sono in genere sembrate allontanarlo da questa. In tal senso, Passioni e Menzogne sembrerebbero far parte dell'universo negativo della Verità. Ma, come al solito, oltrepassata la soglia dell'ovvio, esse possono rivelarsi in modo del tutto diverso o, addirittura, rovesciato, quasi a suggerire che possano proprio esse essere interpretate come costruzioni foriere di verità e conoscenze, se non uniche, certamente altrettanto profonde delle figure ad esse abitualmente contrapposte. Infatti, a tale proposito, alcune brevi annotazioni vanno fatte.

1. La ricerca è finanziata dall'Università degli Studi di Salerno sui fondi ex $60 \%$ per gli anni acc. 2001-2003.

2. Cfr. S. VegetTi Finzi (a cura di), Storia delle passioni, Bari: Laterza, 1995.

3. Cfr. A. Tagliapietra, Filosofia della bugia. Figure della menzogna nella storia del pensiero occidentale, Milano: Mondadori, 2001. 
In primo luogo, la riflessione filosofica, come il mito e la letteratura, ha mostrato a più riprese come Verità e Menzogna siano strettamente intrecciate in quella «doppia relazione» o quel «rapporto di due rapporti» così come definiti da Jankélévitch:

Quanti giudizi avviluppati in una semplice menzogna! La nozione del vero e del suo contrario e la conoscenza della legge che regola il passaggio dall'uno all'altro, tutte queste complicazioni denunciano la coscienza di Ulisse, lo scaltro, malizioso saggio Ulisse polymechanos Odysseus. ${ }^{4}$

in una dimensione che presuppone come la possibilità della menzogna sia data con la coscienza stessa, di cui è misura di grandezza e bassezza:

E come la maturità è simultaneamente la coscienza di sé e della giovinezza, così la coscienza menzognera è una coscienza a fortiori e doppiamente cosciente, dato che essa comprende in sé l'ingenuità superandola. ${ }^{5}$

Allo stesso modo, nella poliformità dei comportamenti e nelle parole degli individui, esse, spesso, acquisiscono identica o, quantomeno, analoga funzione sociale. In tal senso, il riferimento iniziale, che si ritrova nel volume di Tagliapietra, a Socrate e a Jacob il bugiardo è di per sé del tutto trasparente. L'esercizio della parresia — l'esser franco a costo della propria vita — come quello sistematico della menzogna, finiscono, quindi, per assumere, in particolari condizioni pari dignità. D'altronde se Ulisse non è altri che un "portavoce» della divinità nel senso di Foucault, e ciò assicura autorevolezza al suo discorso, Socrate, analogamente, non è che la voce attraverso cui si esprime il codice morale.

In secondo luogo, come ha ben dimostrato Michel Foucault, ${ }^{6}$ le successive complesse e intricate operazioni di «separazione» all'interno dell'universo discorsivo sono sempre storicamente determinate e sono accompagnate da riorganizzazioni non solo e non tanto teoriche, ma realizzate nella pratica sociale delle istituzioni originate: dunque, anche nella vita dei singoli individui e nel loro uso linguistico. Basti pensare al cosiddetto "grande internamento" seguito alla scissione Follia/Ragione in epoca moderna.

In terzo luogo, non soltanto la filosofia contemporanea ha, per così dire, rivalutato l'idea di passione, ma, soprattutto, l'epistemologia e la sociologia della scienza dell'ultimo quarto del secolo trascorso, come gli stessi sviluppi delle scienze cognitive, ${ }^{7}$ hanno messo il luce l'intreccio positivo tra emozione

4. V. JankÉLÉVITCH, Du mensonge, Paris: Flammarion, 1998 (trad. it. La menzogna e il malinteso, Milano: R. Cortina ed., 2000, p. 7.

5. Ibid., p. 7.

6. Cfr. M. Foucault, L'ordre di discours, Paris: Gallimard, 1971 (trad. it. L'ordine del discorso, Torino: Einuadi, 1978).

7. In relazione alla recente riscoperta della normale continuità tra la sfera emozionale e quella cognitiva si veda, in particolare, A. R. DAMASIO, Descartes'Error. Emotion Reason and the Human Brain, London: Papermac, 1994 (trad. it. Lerrore di Cartesio. Emozione, ragione e cervello umano, Milano, Adelphi, 1995), e D. Goleman, Emotional Intelligence, New York: 
e ragione, riflettendo sul legame tra la vita emozionale ed affettiva e quella di ordine razionale e concettuale, quasi riprendendo il senso delle affermazioni della sacerdotessa Diotima nel Simposio di Platone, per la quale non c'è filosofia (conoscenza) senza desiderio. Qui ricorderò, come esempio particolare, che il ruolo giocato dalle emozioni in relazione alla memoria è stato sottolineato, prima ancora che in epoca moderna, già in epoca classica da Cicerone in relazione alle mnemo-tecniche. Nella Retorica ad Erennio, infatti, si può leggere:

Imagines igitur nos in eo genere constituere oportebit quod genus in memoria diutissime potest haerere. Id accidet si quam maxime notatas similitudines constituemus; si non multas nec vagas, sed aliquid agentes imagines ponemus... ${ }^{8}$

D'altronde, all'atto stesso di quella che è stata più volte riconosciuta come l'ennesima ferita arrecata all'auto-coscienza dell'umanità, e cioè all'atto stesso della scoperta freudiana dell'inconscio, cioè del rinvenimento di un nuovo determinante vincolo alla pretesa libertà degli individui, è stata favorita la comprensione della limitatezza della pretesa autonomia dei piani della Ragione e della Verità come categorie universali regolate unicamente da «regole» e «confutazioni». In tal senso, quella che può essere definita, a mio avviso, la «creativa spietatezza» dell'epistemologia deterministica di Feyerabend ricorda, a chi ha come mestiere la pratica di una qualsiasi riflessione teorica, come la conoscenza sia il frutto della "partecipazione» e che:

Il modo in cui i problemi scientifici vengono affrontati e risolti dipende dalle circostanze in cui tali problemi sorgono, dai mezzi (formali, sperimentali, ideologici) disponibili e dai desideri di coloro che li affrontano. Non vi sono immutabili condizioni al contorno della ricerca scientifica. ${ }^{\text {? }}$

Comunemente temi come quelli scelti sembrano essere campi da gioco per squadre composte da filosofi, letterati, psicologi, antropologi, semiologi e sociologi della comunicazione. In realtà, i linguisti che, oltre che dal possesso di tecniche vieppiù elaborate e formalizzate, siano spinti da una vocazione intellettuale alla comprensione delle ragioni del loro "mestiere» e del senso complessivo del proprio oggetto di studio - le lingue storico-naturali - si collocano, in un certo senso, in una dimensione che costituisce un osservatorio particolare e privilegiato cui non è possibile rinunciare. Essi, infatti, si trovano in una condizione specialmente favorita, cioè posseggono la strumentazione necessaria per descrivere minuziosamente quanto una lingua mette a disposi-

Bantam, 1995 (trad. it. L’intelligenza emotiva, Milano: Rizzoli, 1996). Per una presentazione generale delle questioni correlate si veda anche C. BAZZANELla e P. KoBaU (a cura di), Passioni, emozioni affetti, Milano: Mc Graw-Hill, 2001.

8. Cicerone, $A d$ C. Herennium libri IV de ratione dicendi (Rhetorica ad Herennium), edizione Harvard Univ. Press 1968, L. III, XXII, 37.

9. P. K. FEYERABEND, Farewell to Reason, London-New York: Verso, 1997 (trad. it. Addio alla ragione, Roma: Armando 1990, p. 300). 
zione dei parlanti quando questi, per i casi delle loro vite, s'imbattono nelle nebbie della bugia, dell'ira, della passione amorosa, di quella politica ecc. Cioè quanto una lingua rende disponibile all'uso comune. Infatti, se Sergio Moravia, nel saggio incluso nel volume citato della Vegetti Finzi, coglie nel segno affermando:

Presto, assai presto, ci accorgiamo che la passione è, in prima approssimazione, una parola, un concetto. E, più esattamente, un costrutto teorico, connesso a matrici e fini plurimi, che l'uomo applica ad una determinata area di vissuto per evidenziarne certi tratti e dar loro un significato, una voce. Se ci si riflette bene, quest'acquisizione è tutt'altro sterile o banale. Dire che la passione è una parola/concetto anziché una cosa implica lo spostamento della nostra indagine dal piano dell'ontologia a quello dell'ermeneutica. Implica sostituire la domanda che cosa è la passione? con la domanda di cosa parla la passione? [...] Secondo questa prospettiva, la passione (beninteso non solo essa) è essenzialmente un grande apparato significante. $\grave{E}$ anche, per riprendere una metafora cara a Richard Rorty, un certo vocabolario. Un vocabolario, si badi, che non viene considerato l'unico in grado di descrivere o giudicare determinati referenti, e che non pretende alcuno statuto privilegiato. Ma purtuttavia un vocabolario che, in determinate circostanze, viene preferito a quelli disponibili. Evidentemente il modo in cui esso dice la nostra esperienza appare meglio rispondente ad esigenze e finalità per noi in quel momento più rilevanti di altre. Un vocabolario, infine, che a sua volta si correla a ben precisi presupposti esterni a quel lessico. ${ }^{10}$

allora, uno dei possibili compiti del linguista è, allora, la definizione di tale "vocabolario» operata a partire dalla propria scelta teorica e dalla batteria di strumenti che questa gli attribuisce. Un vocabolario rortriano, dunque, tra quelli possibili e in alternativa reciproca che si è sostituito a quelli precedenti. ${ }^{11}$

\subsection{La descrizione lessico-grammaticale}

In quest'occasione, quindi, si tratta di un'operazione descrittiva costruita sulle premesse della "grammatica in operatori e argomenti» di Zelig S. Harris e dall'opzione teorico-metodologica di Maurice Gross. Va allora chiarito come s'intenda convertire l'idea di Moravia di «apparato significante» e di «vocabolario» di Rorty in termini harrisiani e in termini lessico-grammaticali.

Se con «apparato significante» s'intende quell'insieme di strumenti aventi, in una lingua storico-naturale, la medesima funzione di manifestare, esprimere un dominio di significato omogeneo, una grammatica harrisiana contempla, in questo caso, l'individuazione delle classi di operatori elementa-

10. S. Moravia, «Emozione e passione», in S. Vegetti FInZI (a cura di), Storia delle passioni, cit., p. 4-5.

11. Il riferimento, in questo caso, è a R. RORTY, Contingency Irony and Solidarity, Cambridge: CUP, 1989 (trad. it. La filosofia dopo la filosofia. Contingenza ironia e solidarietà, Bari: Laterza, 1989), in particolare alla Parte prima. 
ri e non elementari definite da tale campo e delle classi di equivalenze distribuzionali e parafrastiche ad essi associati, cioè quelle classi di frasi possibili correlate in senso trasformazionale.

In tale direzione vale la pena ricordare che, se il problema di definizione delle caratteristiche combinatorie generali di un qualsiasi linguaggio è, in senso formale, quello della separazione delle combinazioni di unità in esso contenute da quelle che non lo sono, allora, tale questione fondamentale si ritrova anche nel caso di una lingua naturale, almeno per quella dimensione puramente calcolistica che in essa si riconosce. Ciò significa che, per Harris, l'indagine sistematica sui vincoli operanti sulle combinazioni possibili è particolarmente significativa:

Tale centralità è indicata dal fatto che ogni vincolo che contribuisce alla struttura della frase offre insieme un contributo costante e definito al significato di quella frase, come pure dal fatto che il primo vincolo crea un ente matematico - formato non da concetti astratti, ma dalle reali occorrenze di parole nelle frasi - come struttura fondamentale delle frasi. Si può osservare che, mentre il quadro della lingua presentato qui può sembrare troppo riduttivo, per il fatto che strutture complesse sono definite in termini di vincoli relativamente semplici, questa non è una visione riduzionista come il ritenere che un sistema sia costituito da nient'altro che dai suoi componenti. La frasalità non è solo la scelta di parola, ma una relazione nuova — dipendenza dalla dipendenza - sulle parole; una frase singola esemplifica non solo la frasalità, ma una relazione di verosimiglianza sulle singole parole dipendenti. ${ }^{12}$

La teoria della sintassi di Harris, nel passaggio dalla fase strettamente distribuzionalista a quella successiva di tipo trasformazionalista, individua, per l'appunto, nella nozione di "trasformazione» la relazione tra due sotto-insiemi di frasi con costante variazione di forma ma in rapporto di parafrasi:

La trasformazione è così una funzione nella quale la variazione di forma opera su ogni frase di un sotto-insieme per produrre («derivare») la corrispondente frase (immagine) nell'altro. La frase e la sua immagine risultano approssimativamente parafrastiche. ${ }^{13}$

12. Z. S. HARRIS, Language and Information, Columbia University Press, 1988 (trad. it. a cura di M. Martinelli, Linguaggio e informazione, Milano: Adelphi, 1995, p. 45).

13. Ibid., p. 23. In realtà, se si applica con regolare sistematicità il principio strutturalista dell'equivalenza distribuzionale, come quello dell'equivalenza grammaticale, così come definito da Harris (cfr. Z. S. HARRIS, Discourse Analisys [1952], in ID., Papers in Structural and Transformational Linguistics Dordrecht: Reidel Publishing Co., 1970), a nostro avviso, ci si rende conto come i termini utilizzati ("produrre» e "derivare») si riferiscano, non tanto ad una concezione della trasformazione come «derivazione» di una frase dall'altra, quanto piuttosto ad una "relazione» tra frasi che nel sistema sono strettamente equivalenti fra loro, cioè sono equi-probabili. Infatti, nel caso delle trasformazioni di tipo unario, ad esempio, nessuna considerazione sistemica consente di dire che le frasi che costituiscono una classe di equivalenze distribuzionali e parafrastiche - cioè una classe di frasi — siano tra loro in un 
Inoltre, va ricordato come l'indagine di tipo harrisiano si concentri sui vincoli operanti sulla combinatoria linguistica e sulle restrizioni che influenzano l'equi-probabilità di occorrenza delle parole l'una rispetto all'altra, individuando tre tipi di vincoli che veicolano ognuno un tipo di significato, in modo tale che si possa ritenere che il significato di una frase sia determinato direttamente dalle parole e dai vincoli: un vincolo di ordine parziale, una distribuzione di probabilità non uniforme e una riduzione di forme fonemiche. In particolare, il primo, quello di ordine parziale, organizza la struttura frasale, agendo tra occorrenze di parole negli enunciati: si tratta di un ordine parziale sulle parole consistente in un ordinamento in cui alcune parole sono più in alto o più in basso rispetto ad altre, mentre altre non sono né più in alto né più in basso tra loro. In pratica, l'ordine parziale costituisce un vincolo sulle combinazioni delle parole: esso dice che nella posizione argomento prossima ad un dato operatore la probabilità di certe parole - quelle che non appartengono alla classe di argomenti per quell'operatore - è zero. In tal senso:

Ora, questa relazione di dipendenza ha una proprietà importante. Se ci chiediamo cosa determina per ogni parola la classe di parole richieste come argomento, troviamo che queste sono identificate da ciò che a loro volta richiedono. Nel nostro campione di parole, parole come man, tree, earth - per la maggior parte nomi concreti - possono essere definite come nomi che richiedono zero, cioè niente. Quindi sleep, fall — cioè per la maggior parte dei verbi che potremmo definire come intransitivi «concreti» - richiedono una parola di quella classe, cioè richiedono una parola che richiede zero. E wear, find verbi transitivi «concreti» richiedono due parole che a loro volta richiedono zero. E entail richiede due parole, ognuna delle quali deve necessariamente richiedere qualche cosa (come assert o entail stesso). Vi sono quindi tre livelli di richiesta che sono veramente indispensabili. Vi deve essere, nella lingua e in ogni frase, almeno un argomento a livello zero che non richiede alcunché, altrimenti non si potrebbe avere alcuna parola nella frase. Vi deve anche essere almeno un operatore di primo livello che richiede solo parole che richiedono zero, perché la semplice occorrenza di parole di livello zero non implica la presenza di nient'altro nella frase: operatori di questo tipo sono sia sleep sia wear. E vi dovrebbero essere operatori di secondo livello, tali che almeno una delle loro richieste sia un operatore di primo livello, affinché vi siano frasi non elementari. Questo è il caso sia di assert sia di entail. ${ }^{14}$

Mentre il primo vincolo delinea l'impalcatura della frase, il secondo - quello della verosimiglianza di occorrenza - illustra in che modo una particolare parola entra a far parte di una frase e per quali motivi certe combinazioni sono più probabili di altre:

rapporto altro da quello stabilito per il loro essere membri della classe, cioè varianti una dell'altra. I membri di una classe di frasi, al pari dei membri di una classe fonologica - gli allofoni - costituiscono semplicemente varianti sulle quali operano, naturalmente, vincoli di natura diversa. 
Intendiamo qui, per verosimiglianza di una parola sotto un operatore (o per un argomento), una stima della probabilità o della frequenza di quella parola rispetto a un fissato numero di occorrenze di quell'operatore (o argomento) ... Ogni parola esercita una selezione alquanto sfumata sulle altre parole che occorrono nella posizione dei suoi argomenti - vale a dire una selezione che rende la distribuzione diversa da quanto ci si aspetterebbe se le occorrenze fossero casuali o con pari frequenza. Sotto sleep, questo vale per centinaia di parole come man e persino tree, in contrasto con earth, raro, oppure stone o universe, ancora più rari. L'insieme delle parole che hanno frequenza più alta della media è chiamato la selezione. Il significato principale di una parola è dato dal significato della selezione degli argomenti su cui opera o della selezione degli operatori di cui è argomento ... Per esempio, nel caso dei pronomi indefiniti something, someone, pressoché ogni operatore nella lingua accetterà facilmente l'uno o l'altro di questi come argomento. Quindi la loro verosimiglianza totale è alta ... Sotto and e but è più probabile che alcune parole in una data posizione della seconda frase siano le stesse di quelle nella corrispondente posizione della prima frase rispetto alla possibilità che tutte le parole siano diverse. ${ }^{15}$

Infine, va ricordato come, in termini di analisi lessico-grammaticale, si tratti di individuare la relazione sistematica tra elementi lessicali e insieme delle forme grammaticali di una lingua attraverso l'analisi dei vincoli, delle restrizioni, operanti su di essa, cioè si tratti di costruire una grammatica lessicalmente esaustiva.

Tale è l'obiettivo descrittivo che l'indagine cui questo contributo si riferisce si pone. Individuare cioè la grammatica lessicalmente esaustiva dell'italiano associata alla tradizionale "figura del male» della Menzogna, descrivendo, quindi, le classi di strumenti linguistici che l'italiano comune rende disponibili all'uso dei parlanti grazie alla metodologia di rappresentazione impiegata, a partire da Gross, ${ }^{16}$ nei lavori di Elia, Martinelli e D’Agostino. ${ }^{17}$

\subsection{La coscienza menzognera}

Anche se una situazione di questo tipo non ha nulla di usuale, è possibile immaginare che due bastardini che non si conoscono, in un ascensore che va verso il settimo piano, dopo qualche attimo di perplessità dovuto all'ambiente, inizierebbero a fiutarsi e a strusciarsi l'uno contro l'altro e, alla fine del breve viaggio, ognuno andrebbe per la sua strada. Se al loro posto, invece, si trovassero, come è più comune, due esemplari della specie umana, è molto probabile che, scartati normalmente per ovvie ragioni odorato e contatti fisici, almeno uno dei due sconosciuti non riuscirebbe a resistere alla tentazione di aprir bocca. In quei pochi secondi che separano il percorso pianoterra-settimo piano,

15. Ibid., p. 33-34.

16. Cfr. M. Gross, Méthodes en syntaxe, Paris: Hermann, 1975.

17. Cfr. A. Elia, M. Martinelli, E. D'Agostino, Lessico e strutture sintattiche. Introduzione alla sintassi del verbo, Napoli: Liguori, 1981; e E. D’Agostino, Analisi del discorso. Metodi descrittivi dell'italiano d'uso, Napoli: Loffredo, 1992. 
quella micro-conversazione priva di contenuto informativo reale manifesta il fatto che l'uso del linguaggio verbale è fondamentale modalità della socialità umana, cioè strumento a disposizione per avere una qualsivoglia forma di relazione con gli altri. Parliamo con tutti, indipendentemente dal grado di conoscenza e familiarità. Con i tassisti, le bariste, i compagni di treno o di metropolitana, con la signora che è attenta a osservare la stessa vetrina. E, immediatamente dopo, ognuno per la propria strada, così come i due bastardini. Infatti, è quantomeno molto improbabile che, al termine di qualche scambio di battute, prendano inizio impreviste avventure. Nella maggior parte dei casi, così, si parla tanto per parlare. Si potrebbe anche dire che ciò «serve alla coesione sociale o di gruppo", ma sarebbe già un'affermazione molto forte. Ma è sempre, comunque, inopportuno assolutizzare le affermazioni procedenti da osservazioni empiriche e, infatti, l'antropologia, la linguistica e la sociolinguistica hanno mostrato come esistano comunità nelle quali parlare, ad esempio, sia a sconosciuti, come a persone ben note, in particolari circostanze, non sia affatto ritenuto un comportamento autorizzato e legittimo, anzi possa essere del tutto disdicevole. E non bisogna forzosamente richiamarsi a comunità indo-americane o per noi esotiche, basta recarsi in qualsiasi paesino dell'Italia meridionale. ${ }^{18}$ Parliamo anche da soli, comunque, sia in situazioni legittimate sia in situazioni che non lo sono affatto, o ci sentiamo in obbligo, dopo essere inciampati camminando, di "far ripartire il sistema» e di "recuperare la nostra credibilità» esclamando qualcosa, come ha rilevato Goffman. ${ }^{19}$ Naturalmente, parliamo (o scriviamo), e con ciò "facciamo» o "agiamo», in una gamma di situazioni e per una gamma di scopi estremamente ricca e articolata all'interno tale che, molto spesso, le relative sistematizzazioni e classificazioni appaiono semplificazioni e riduzioni adeguate soltanto alle filosofie e alle teorie che le hanno generate. È possibile, infatti, ritenere che domande come «quando parliamo (o scriviamo) che cosa facciamo?», oppure "quando parliamo (o scriviamo) di cosa parliamo (o scriviamo)?», oppure ancora "quando parliamo (o scriviamo) perché parliamo (o scriviamo)?" non abbiano risposte soddisfacenti e che, in realtà, a tali interrogativi potrebbero rispondere soltanto i parlanti, a condizione di essere in grado di osservarsi ironicamente. Non sembra affatto facile ed è possibile che ci siano riusciti soltanto alcuni che, più che in scritture filosofiche o scientifiche, si siano impegnati in scritture letterarie. In ogni caso, si potrebbe dire molto semplicemente che il parlare è il nostro "pane quotidiano».

Se restringiamo sufficientemente il campo dei "perché» e dei "cosa», certamente possiamo pensare che, in qualche caso, si parli per affermare o difen-

18. Si veda a tale riguardo K. H. BASSO, «To give up on words: silence in western Apache culture», Southwestern Journal of Antrhropology, XXVI, n. 3, 1970, p. 213-230 (trad. it. "Il silenzio nella cultura degli Apache occidentali» in P. P. GIGLIOLI (a cura di), Linguaggio e società, Bologna: il Mulino, 1972).

19. Si veda a tale proposito E. GofFMAn, Forms of Talk, Philadelphia: Univ. of Pennsylvania, 1981 (trad. it. Forme del parlare, Bologna: il Mulino, 1987), in particolare il saggio Gridi di reazione. 
dere la/una verità oppure, all'inverso, per occultarla, manipolarla, oppure, addirittura, per modificarla. Per tessere, attraverso grandi menzogne o piccole bugie, inganni, tradimenti, cospirazioni, ribaltoni, truffe, giochi delle tre carte. Oppure, ancora, per adattare comodamente la verità alle nostre debolezze. Non tutti sono Socrate e affermare la verità a costo della propria vita è stata ed è una virtù - o un vizio - certamente - o fortunatamente — raro. ${ }^{20}$ Oltretutto, la prudenza insegna a diffidare sempre di coloro che affermano la propria incapacità a parlare se non in termini, con parole di verità. ${ }^{21}$

Se è pur vero che non c'è bisogno delle parole per mostrarsi sinceri o insinceri e che, dunque, verità e menzogna ci parlano anche soltanto con gesti e comportamenti e se è pur vero anche che, in modo del tutto involontario, gesti, comportamenti e parole possono indurre in inganno, come dimostrano frasi a costruzione causativa del tipo:

\section{il fatto che Eva non rispondesse $(\mathrm{E}+$ volontariamente + involontariamente) ha tratto in inganno Luca}

Eva ha ingannato Luca $(\mathrm{E}+$ volontariamente + involontariamente $)$

contraddistinte, in lingue come l'italiano, da una duplicità di interpretazione associata al tratto ( \pm volontario); è certamente altresì vero che una parte cospicua del nostro parlare (e scrivere) è volto coscientemente e volontariamente alla manipolazione delle informazioni, o dei loro processi di acquisizione da parte dei nostri interlocutori, cioè: è finalizzato alla menzogna. Cioè alla produzione di enunciati che un osservatore esterno potrebbe classificare in forme di frase come:

Ugo mente a Maria

Ugo è un mentitore

Ugo è menzognero

Che F è una menzogna di Ugo 22

20. Per la questione della franchezza e dell'affermazione della verità nella Grecia antica si veda M. Detienne, Les maîtres de vérité dans la Grèce arcaïque, Paris: Maspero, 1967 (trad. it. I maestri di verità nella Grecia antica, Bari: Laterza, 1977); e M. FouCAULT, Discourse and Truth. The Problematization of Parrhesia, Evanston: Nothstern University Press, 1985 (trad. it. Discorso e verità nella Grecia antica, Roma: Donzelli ed., 1996).

21. Valga per tutti l'esempio dell'orazione di Antonio nel Giulio Cesare di Shakespeare che normalmente è considerata un modello di retorica politica: «Non son venuto, amici, a rapire per me il vostro cuore; non sono un oratore come Bruto, sono - mi conoscete - un uomo semplice che amava Cesare con cuor sincero; e questo sanno bene anche coloro che m'han concesso il loro beneplacito a parlare di lui così, in pubblico; perché io non posseggo né l'ingegno, né la facondia, né l'abilità, né il gesto, né l'accento, né la forza della parola adatta a riscaldare il sangue della gente: parlo come mi viene sulla bocca, vi dico ciò che voi stessi sapete, vi mostro le ferite del buon Cesare, povere bocche mute, e chiedo a loro di parlar per me. S’io fossi Bruto e Bruto fosse Antonio, allora sì, che qui a parlare a voi vi sarebbe un Antonio ben capace di riscaldare gli animi e di dar voce ad ogni sua ferita per trascinare a Roma anche le pietre alla rivolta ed all'insurrezione!» (atto III, sc. II).

22. F è costituita da una soggettiva sulla quale non operano particolari restrizioni di selezione. 
che si prestano ad un'interpretazione per la quale a $X$ si associa con maggiore probabilità il tratto (+ volontario).

Come s'è detto, alle classi di equivalenze parafrastiche e distribuzionali associate al campo della menzogna e dell'inganno è dedicato questo contributo. Non ci si occuperà della secolare disputa sulla Verità o sulla natura e sulle caratteristiche degli enunciati veri o falsi, ma di ciò che l'italiano d'uso comune mette a disposizione dei parlanti quando si avvicinano al problema della menzogna e dell'inganno.

\subsection{Un mondo di menzogne, inganni e sotterfugi}

A quanto pare gli uomini e le donne mentono di continuo, con un grado maggiore o minore di consapevolezza e di sfrontataggine e con finalità molto diverse. Con Zani, Selleri e David, ${ }^{23}$ si ricorderà che, nell'uso comune e quotidiano di una lingua, il numero degli enunciati che, per ragioni diverse, sono riconosciuti (dai loro stessi emittenti) come pienamente "veritieri» rappresentano una percentuale relativamente limitata. Zani, Selleri e David citano la ricerca di Adler e Towne, ${ }^{24}$ per i quali, con un campione di 130 soggetti, solo il 38,5\% delle frasi in situazione di conversazione rientrava in tale classe. Per una visione d'insieme delle teorie comunicazionali sulla menzogna rimando a Anolli, ${ }^{25}$ in particolare per l'Information Manipulation Theory e l'Interpersonal Deception Theory.

D'altronde ciò è noto all'esperienza comune. Dalle televendite, ai patti e "contratti» in politica, alle promesse degli amanti, alle "bugie a fin di bene», alle piccole omissioni e distorsioni di quello che si ritiene essere la verità, il nostro mondo è apparso sempre in rapporto con la menzogna e l'inganno. Un numero elevatissimo di enunciati e frasi, siano essi legati a previsioni improbabili o impossibili sul futuro, o al gusto dell'iperbole che spessissimo ci contagia, o siano decisamente votati alla falsità:

\section{le automobili tedesche durano tutta la vita ti amerò sempre creeremo tre milioni di posti di lavoro questo sformato è eccezionale la sogliola è freschissima Saddam ha come bersaglio anche l'Italia $i$ sondaggi continuano a darci al 49\%}

appaiono ad un normale osservatore disinteressato come potenzialmente più o meno menzogneri e capaci, a certe condizioni, di trarre in inganno chi li ascol-

23. Cfr. B. Zani, P. Selleri, D. David, La comunicazione. Modelli Teorici e contesti sociali, Roma: NIS, 1994.

24. Cfr. R. B. Adler, N. Towne, Looking out Looking in, London: Holt Rinehart and Winston, 1990.

25. Cfr. L. Anolli (a cura di), Psicologia della comunicazione, Bologna: il Mulino, 2002. 
ta. Infatti, non è certamente un caso che menzogna ed inganno siano anche oggetto di una diffusa letteratura scientifica e di divulgazione: oggi non più esclusivamente tema di filosofi — dal paradosso del mentitore in poi — o di teologi e di Padri della Chiesa, ma anche di sociologi, psicologi ecc. ecc. ecc. Tema di confine: dalla teoria della seduzione come apparenza - quindi come inganno - a quella della creazione letteraria — quindi come non-verità fattuale - a quella dell'evoluzione del cervello umano e a quella dello sviluppo cognitivo.

In realtà, tale ipertrofia della riflessione su menzogna e inganno si fonda, probabilmente, sulla coscienza che gli uomini vivono in un universo che per molti aspetti manifesta di continuo un carattere ingannevole e che i luoghi della menzogna sono molteplici. Se il Serpente ha ingannato Eva e, quindi, Adamo, stando a quanto hanno rilevato naturalisti, etologi e socio-biologi, il regno della Natura appare esso stesso contraddistinto dalla presenza di comportamenti e processi ingannevoli: camaleonti, lucciole, piante carnivore e fiori, tutti, senza distinzione di sorta si servono di inganni, in una dimensione di lotta per la vita e di evoluzione. Un dominio, questo naturale, dominato, dal "gene egoista» di Dawkins. ${ }^{26}$ Sommer, nel ripercorrere la storia dei modelli di interpretazione di questi comportamenti animali, elenca una lunga e, per certi aspetti sorprendente, casistica di comportamenti ingannevoli registrati in natura che spingono a ritenere che siano proprio le bugie ad avere le gambe lunghe, tanto che si può sostenere con l'autore che:

la funzione naturale della comunicazione in sostanza non risiede affatto nel trasmettere un'informazione veritiera. ${ }^{27}$

Se, nel campionato della bugia di scimmie e primati antropoidi, gli scimpanzè e i bonobo, per altro strettamente imparentati filogeneticamente tra loro, si collocano al primo posto, classificati meglio di gorilla, oranghi e gibboni, e se, nello sviluppo delle varie fasi dell'intelligenza senso-motoria nei bambini un possibile, anche se sempre difficile, parallelismo tra bambini e piccoli di scimmie di specie diverse è registrabile solo in parte, per tempi e fasi raggiunte, è comunque da mettere in evidenza che nel cosiddetto «sesto stadio» di sviluppo (dall'anno e mezzo ai due anni) dei bambini ci sono tutte le condizioni che caratterizzano il cosiddetto «inganno tattico»:

ciò che conta è raffigurarsi mentalmente un obiettivo, per poi riuscire a manipolare subito, fin dal primo tentativo, il destinatario di un'informazione al fine di spingerlo nella direzione desiderata. ${ }^{28}$

26. Cfr. R. DawkIns, The Selfish Gene, Oxford University Press, 1976 (trad. it. Il gene egoista, Milano: A. Mondadori, 1989).

27. V. Sommer, Lob der Lüge. Täuschhung und Selbstbetrug bei Tier und Mensch, München: Beck's Verlag, 1992 (trad. it. Elogio della menzogna. Per una storia naturale dell'inganno, Torino: Bollati Boringhieri 1999, p. 65).

28. Ibid., p. 137. 
Ciò implica, nonostante tutte le difficoltà, che non solo uomini e primati ingannino alla stessa stregua, ma, e ciò è più importate, che menzogna ed inganno siano per definizione nello stesso patrimonio genetico degli uomini, cioè nascano in pratica con essi e accompagnino come specie. Lo stesso problema della "coscienza» in uomini e specie animali potrebbe, in tal senso, essere ribaltato: non più la serie di interrogativi del tipo «hanno alcuni animali una forma di coscienza equivalente a quella degli esseri umani?», ma piuttosto interrogativi del tipo «posti dinanzi alla scelta tra ingannare e non ingannare, gli uomini ed alcuni animali hanno le stesse possibilità di optare per la seconda alternativa?». In ogni caso, la consapevolezza, in questo caso, dello stretto legame tra il comportamento umano e comportamento animale, ci fa rileggere, sorridendo, il testo di Da Ponte per Le nozze di Figaro mozartiano a proposito delle donne:

Aprite un po' quegli occhi uomini incauti e sciocchi. Guardate queste femmine Guardate cosa son. Queste chiamate Dee. Dagli ingannati sensi, a cui tributa incensi la debole ragion. Son streghe che incantano Per farci penar, sirene che cantano per farci affogar; civette che allettano per trarci le piume, comete che brillano per toglierci il lume. Son rose spinose, son volpi vezzose, son orse benigne, colombe maligne, maestre d'inganni. Amiche d'affanni Che fingono, mentono, che amore non sentono, non senton pietà. Il resto nol dico, già ognuno lo sa. (atto IV)

Se la nostra vita ci ha visto attuare anche come ingannatori e bugiardi e se ingannatori e bugiardi lo sono stati, lo sono e lo saranno tutti o quasi, certamente alcuni hanno meritato una fama particolare, tanto da rimanere nella nostra memoria fondamentalmente come figure della menzogna e dell'inganno. Ognuno di loro con caratteri e con obiettivi diversi, con tratti che ci permettono di identificarli bene. Certamente Achille, il semidio, mente anch'egli, ma non lo si ricorda per questo, ma per la sua epica collera o per il suo troppo umano tallone; Ulisse, l'uomo, è certamente anch'egli un eroe epico, ma, per la sua metis, rientra nella nostra mitologia come «l'ingannatore»e «il mentitore» per eccellenza. Alcuni più di altri, dunque, hanno posseduto il carattere di tessitori di inganni. Se la storia strictu sensu dell'umanità inizia con l'inganno del serpente, mentiranno Prometeo, Marco Antonio, Iago, Don Giovanni, Jakob. Dopo che il tradimento di Giuda, compiuto perché si compisse il disegno di Dio, ha mentito Simon Pietro. Hanno mentito e continueranno a farlo gli scienziati. ${ }^{29} \mathrm{La}$ coscienza di ciò renderà il mendacio il "peccato di lingua» più importante e diffuso, secondo soltanto al profferire il vero nome di Adonài, el Eloìm, o alla bestemmia:

\section{Salmi, Libro I (1-41)}

Salmo 4 Preghiera della sera

3 O uomini, fino a quando disprezzerete la mia gloria amerete il nulla e cercherete la menzogna? 
$[\ldots]$

Salmo 5 Preghiera del mattino

6/7 Tu respingi chi opera il male

fai perire i bugiardi,

l'uomo di violenza e d'inganno

Adonai/YHWH lo detesta.

[...]

nella loro bocca non c'è sincerità

il loro ventre è delitto

la loro gola è tomba divorante

la loro lingua seduce. ${ }^{30}$

\subsection{Una definizione}

A questo punto, però, appare necessario introdurre qualche definizione da cui partire. In un normale dizionario d'uso dell'italiano, di menzogna viene fornita una definizione come questa:

affermazione contraria a ciò che è o si crede corrispondente a verità, pronunciata con l'intenzione di ingannare e con fini malvagi o utilitaristici; volontaria deformazione, deliberato travisamento del vero: una menzogna ignobile, sfacciata, spudorata; un discorso pieno di menzogne, menzogna pia, pietosa, detta a fin di bene; letterario: avere faccia, sembianza di menzogna, presentarsi con i tratti inconfondibili del falso: una verità che ha, troppo più di quello che ella fu, di menzogna sembianza (Boccaccio) ${ }^{31}$

mentre, in particolare, nella letteratura comunicazionale, «menzogna» ed «inganno» sono stati spesso etichettati come manifestazioni dei fallimenti della comunicazione umana e, molto spesso, collocati in una dimensione «morale». ${ }^{32}$ Zani-Selleri-David (1994), ad esempio, sostengono:

30. Per quanto riguarda l'Antico Testamento la questione della menzogna appare in vari contesti. La forma $k z b$ indica primariamente la menzogna (verbale) ed è possibile distinguere nel contesto del diritto lo spergiuro, la falsa testimonianza e la calunnia, mentre nel contesto specificamente religioso si riferisce all'infedeltà nei confronti di Dio, alla devozione agli idoli ingannatori e nella falsa profezia. Per quanto riguarda il Nuovo Testamento in Paolo si ritrova la forma del verbo greco pseúdomai ed il sostantivo pseudos che indica la condotta dell'umanità peccatrice che ha scambiato la verità di Dio con la menzogna. A tale proposito si veda il Grande lessico del Nuovo Testamento, Brescia: Paideia, 1988.

31. Cfr. Il grande dizionario della lingua italiana Paravia di De Mauro (2000).

32. Per quest'occasione non si affronterà la dimensione non morale, "creativa», e della cosiddetta "scoperta dell'inutile", spesso invocata da diversi autori. Ci si limita, quindi, a rimandare, oltre al già citato Tagliapietra, a F. NIETZSCHE, Su verità e menzogna in senso extra-morale in ID., La filosofia nell'epoca tragica dei Greci. Escritti 1870-1873, trad. it. a cura di G. Colli e M. Montinari, Milano: Adelphi, 1991, a O. WILDE, "The Decay of Lying», The Nineteenth Century, 1889, ripubblicato in ID., Intentions, 1891 (trad. it. La decadenza della menzogna, Milano: Mondadori, 1995) e a G. BATAILlE, La limite de l'utile (fragments), Paris: Gallimard, 1976 (trad. it. Il limite dell'utile, Milano: Adelphi, 2000). 
In genere, si intende che la comunicazione problematica non sia voluta, ma accada indipendentemente dalla volontà esplicita dei partecipanti per una serie di motivi diversi. Quando invece la miscommunication è intenzionale, allora si ha l'inganno. ${ }^{33}$

Sia nella prima come nella seconda definizione, dunque, appare come pertinente l'intenzionalità e, dunque, il riferimento a quel tratto (+volontario) associabile alle forme di frase in precedenza citate. Associata alla sua generalizzata condanna, la menzogna resta legata, nella dimensione morale, alla definizione di Agostino, nel De Mendacio (395):

Non enim qui falsum dicit mentitur si credit aut opinatur verum esse quod dicit. [...] Quisquis autem hoc enuntiat quod vel creditu manimo vel opinatum tenet, etiamsi falsum sit, non mentitur. Hoc enim debet enuntiationis suae fidei ut illud per eam proferat quod animo tenet et sic habet ut profert. Nec ideo tamen sine vitio est, quamvis non mentiatur, sit aut non credenda credit, aut quod ignorat nosse se putat, etiamsi verum sit; incognitum enim habet pro cognito.

Quapropter ille mentitur qui aliud habet in animo et aliud verbis vel quibuslibet significationibus enuntiat. Unde enim duplex cor dicitur esse mentientis, id est, duplex cogitatio: una rei hujus quam veram esse vel scit vel putat et non profert, altera ejus reiquam pro ista profert sciens falsam esse vel putans. ${ }^{34}$

L'adozione, anche in quest'occasione, della definizione di Agostino riduce ulteriormente l'oggetto d'indagine linguistica in questione, poiché esclude tutte le manifestazioni altre da quelle che appartengono al dominio della coscienza e della volontarietà, del cor duplex e della voluntas fallendi. Restano escluse, pertanto, non solo quelle «menzogne» della natura prima citate, ma anche quelle che, per certi aspetti, parrebbero pur significative per l'occasione, proprio perché mediate per definizione dal linguaggio. Il riferimento è naturalmente a quella classe di "cortocircuiti» della coscienza che si manifestano nella teoria freudiana del «lapsus». ${ }^{35}$ Infatti, se per animali, piante e insetti, una discussione sulla "coscienza" non sembra giustificata e, per i soli mammiferi comunque quantomeno assai problematica, nel caso dei classici lapsus dell'indagine freudiana, è possibile ritenere che l'inganno, la menzogna, consista nella realizzazione non controllata di un apparente «inatteso» per definizione sottostante al livello della coscienza.

33. ZANi, SElleri e DaVID, op. cit., p. 113.

34. Agostino, De mendacio (trad. it. a cura di M. Bettetini, Sulla Bugia, Milano: Bompiani, 2001, p. 31).

35. La definizione di Agostino legata alla volontarietà, come tutte quelle da essa derivate, esclude di fatto dal campo della menzogna il cosiddetto «auto-inganno» e lo assimila al puro «errore», nel senso che l'interpretazione fallace o forzata di segni è frutto di non conoscenza, oppure di un "pre-giudizio" errato che la condizionano. Se, quindi, si abbandona il campo ristretto dell'errore per non conoscenza, l'auto-inganno appare collocabile piuttosto nella dimensione freudiana dell'inconscio. 
In qualche misura, sia la prima (il Dizionario) come la seconda definizione (Agostino) forniscono una rappresentazione della miscommunication «intenzionale» come quella propria di Jankélévitch che, a proposito della "coscienza menzognera» parla, come s'è visto all'inizio, di «doppia relazione» $\mathrm{o}$ "rapporto di due rapporti». Jankélévitch, dall'interno di una dimensione strettamente morale, sembra delineare i tratti caratteristici della coppia Menzogna-Inganno. Oltre a quella che è stata già presentata come la «doppia relazione», in quella «fenomenologia del quotidiano» rappresentata dal breve saggio del 1998, Jankélévitch enumera i tratti della menzogna che appaiono definizionali. La volontarietà e la coscienza della infrazione alla regola, in primo luogo, poiché il mentitore sa bene quel che fa:

Ecco perché la premeditazione non solo non attenua la nostra responsabilità ma ne è una circostanza aggravante: il colpevole cosciente è due volte colpevole, in primo luogo come autore e in secondo luogo come cosciente, essendo la coscienza nel vizio un vizio in più. In ogni caso, se il peccato a differenza dell'errore, è qualcosa che si commette a bella posta, la menzogna diviene per definizione il peccato kat'exochen, non necessariamente il più grave, ma il più caratteristico; fa parte della quintessenza del peccato. Difatti non si mente mai senza volerlo. ${ }^{36}$

Alla coscienza e volontarietà si aggiunge il carattere di peccato fondamentale nella nostra tradizione vetero- e nuovo-testamentaria. In secondo luogo, il carattere legato alla non necessaria identificabilità tra la menzogna e il dire la non-verità:

ciò che definisce la menzogna è una disposizione di coscienza, e non il fatto esteriore, epifenomenico, di dire la non-verità; animi sententia, come dice Agostino, e non rerun ipsarum veritas aut falsitas; il suo caso non è differente da quello dell'intenzione comica, pornografica e umiliante, le quali diventano impalpabili nel momento in cui si vuole assegnar loro un referente oggettivo. ${ }^{37}$

In terzo luogo, il suo essere obbligatoriamente correlata ad un tratto relazionale:

La ragion sufficiente che fa della menzogna un inganno, ossia un'induzione in falsità, è il contesto sociale o più esattamente (dal momento che il due è già un plurale elementare) la presenza dell'Altro; basta la presenza di un io e di un tu perché, senza influsso diretto, si sviluppi una certa corrente come risultato della sola compresenza. È il testimone invisibile e virtuale, il cui sguardo mi impone la prima tensione del faccia a faccia, il primo pudico imbarazzo — "cè qualcuno dietro questa tappezzeria»; è il bellimbusto indiscreto davanti al quale la civettuola si compone. ${ }^{38}$ 
$\mathrm{Al}$ pari degli scimpanzè e dei bonobo già citati da Sommer, gli uomini vivono in uno scenario di fusione-fissione che finisce per definirli, per l'appunto, in termini puramente relazionali, cioè sociali. Tale caratteristica, questione dell'autoinganno a parte, appare come legata in modo fondante alla dimensione comunicativa ed alla possibilità di trasmissione manipolata di informazioni e, in tal senso, è possibile ritenere che in una visione morale della menzogna questa rappresenti un delitto doppio: verso la Verità e l'Innocenza e verso gli altri. In quarto luogo, se il mondo in cui la menzogna vive è un mondo di relazioni, questo non potrà che avere come nuova caratteristica quella dell'opacità. Infatti, si può dire che:

Non si può ingannare colui che sonda le reni e il cuore. La menzogna trova invece la sua ragion d'essere in un mondo di creature parziali, opache, incomunicabili e segrete l'una per l'altra. Per dirla tutta, la menzogna è una strategia destinata a pacificare l'alternativa. La sua vera origine consiste nella rivalità competitiva, in altre parole nell'incapacità delle persone a coesistere uno eodemque loco. ${ }^{39}$

Opacità e vincoli sulla possibilità di comprensione: il dubbio sistematico dello scettico può essere messo in discussione nuovamente e paradossalmente proprio dalla coscienza della non-trasparenza e delle difficoltà che essa mette in atto. Ma se essere sinceri ha un costo - e di Socrate sappiamo - anche non esserlo lo ha, poiché, come quinta caratteristica, la menzogna presenta quella dell' insonnia e della solitudine. Infatti in quinto luogo, si può ritenere che:

La menzogna [...] è insonne e in stato d'allerta: le sue costruzioni non esistono e quindi bisogna confermarle continuamente e difenderle contro le smentite del reale mediante un'autentica creazione continua; un momento di disattenzione ed ecco crollare il castello di carte [...] La menzogna è oberata non solo dalla sua inerzia e precarietà, ma anche dalla solitudine in cui si confina da sé. La vera punizione dei ciarlatani è la perdita della loro ipseità: dal momento che essi non sono né ciò che sono e che seppelliscono nel silenzio, né ciò che gli altri credono che essi siano e che in realtà sono solo per truffa, bisogna concludere che essi non sono più niente. ${ }^{40}$

Infine, come altro carattere, in quinto luogo, la menzogna ha quello di associarsi ad un fantasma, ad una "anima in pena»:

La menzogna, che piega o devia la nostra fede verso i suoi fini interessati, consiste dunque letteralmente nel furto della fiducia; vuole suggerire o fra credere non ciò che pensa ma, come la tautegoria, ciò che dice. Ne deriva che non c'è possibilità di comunione nella menzogna. ${ }^{41}$ 
Ancora una volta ed in modo conclusivo, la menzogna si definisce e sperimenta come negazione di ogni comunicazione: nulla, né il silenzio, né l'assenza che comunque parlano di continuo, è un antagonista tanto poderoso della comunione, perché furto della fiducia, e si presenta come comunicazione fasulla, mascherata. Attraverso un dire, testimonia il non-dire. Attraverso un atto di comunicazione nega la comunicazione stessa. Jankélévitch, non discostandosi da Agostino, in modo sintetico, stabilisce i confini della nostra discussione. ${ }^{42}$

In realtà, quali che siano le ragione particolari di ogni mentire individuale, è possibile ritenere che lo scopo fondamentale della menzogna sia quello di condizionare il comportamento dei nostri possibili interlocutori, traendone da ciò un beneficio, ostacolando e manipolando la loro possibilità di accesso alle informazioni. In tal senso, può farsi rientrare la menzogna nei comportamenti «volti al successo" di cui parla Habermas, come comportamento opposto a quello «orientato all'intesa»:

È possibile invece differenziare le azioni sociali a seconda che i partecipanti assumano un atteggiamento orientato al successo oppure all'intesa; e precisamente tali atteggiamenti devono risultare identificabili nelle circostanze adatte in base al sapere intuitivo degli stessi partecipanti. È quindi anzitutto necessaria un'analisi concettuale di entrambi gli atteggiamenti. Nel quadro di una teoria dell'azione ciò non può essere concepito come un compito psicologico. Non mi propongo come obiettivo di caratterizzare sul piano empirico le disposizioni comportamentali, bensì di cogliere strutture generali di processi di intesa dai quali si possono dedurre condizioni di partecipazione caratterizzabili formalmente. Per spiegare che cosa intendo per atteggiamento orientato all'intesa, devo analizzare il concetto di Verständigung. Non si tratta dei predicati che un osservatore usa quando descrive processi di intesa, bensì del sapere pre-teoretico di parlanti competenti che sono essi stessi in grado di distinguere intuitivamente quando esercitano pressione su altri e quando si intendono con essi e che sanno quando falliscono i loro tentativi.[...] Verständigung è un processo di convergenza tra soggetti capaci di linguaggio e di azione.[...] Un'intesa raggiunta comunicativamente ha un fondamento razionale: non può essere imposta da nessuna parte, sia strumentalmente con l'intervento diretto nella situazione d'azione, sia strategicamente, tramite l'influenza calcolata in vista del successo sulle decisioni di un antagonista. Un'intesa può essere, certo, soggettivamente estorta; ma ciò che accade visibilmente tramite un'influenza esterna o il ricorso alla forza non può contare soggettivamente come intesa.

42. C'è un solo punto sul quale si può dissentire da Jankélévitch, ma è in qualche misura marginale: la questione dell'esclusione della litote dalle manifestazioni della menzogna. Alla sua affermazione per la quale "la litote non è menzognera, ma si propone, al contrario, di indurci alla verità attraverso la via indiretta della simulazione» (p. 5), in realtà si può obiettare che essa può perfettamente essere, invece, strumento della manipolazione menzognera. Infatti, non a caso, lo stesso Iago, che alla fine è riconosciuto come tessitore di inganni e menzogne («Emilia: Hai detto una menzogna, una sporca,maledetta menzogna; sull’anima mia, una menzogna, un'infame menzogna!» Otello, Atto V, scena II), ha per l'appunto tra i suoi strumenti preferiti per l'appunto la litote, «ironia di dissimulazione» per Lausberg. 
Quest'ultima si fonda su convincimenti comuni. L'atto linguistico dell'uno riesce soltanto se l'altro accetta l'offerta in esso contenuta prendendo posizione (sia pure implicitamente) con un sì o con un no su pretese di validità, criticabili in linea di principio. ${ }^{43}$

In tale prospettiva: «la maggiore infrazione del dovere dell'uomo verso se stesso», per dirla con Kant, ${ }^{44}$ cioè la menzogna, non è legata esclusivamente ad «implicature convenzionali», legate cioè al valore delle parole pronunciate dal parlante, ma può altresì legarsi a quelle che Grice ${ }^{45}$ definisce «implicature conversazionali» dipendenti non dalle parole usate, ma da caratteristiche generali del discorso, ogni qualvolta si violi il "principio di cooperazione», per volontà ingannatrice o per volontà esplicita di non cooperazione. Se il principio griciano citato è così formulato:

il tuo contributo alla conversazione sia tale quale è richiesto, allo stadio in cui avviene, dallo scopo o orientamento accettato dello scambio linguistico in cui sei impegnato

e ad esso si associano le note categorie di Quantità, Qualità, relazione e Modo, allora, a quei discorsi che ad un osservatore esterno appaiano dichiaratamente menzogneri vanno aggiunti senza esitazione: omissioni, parziali verità e silenzi.

\section{II}

\subsection{La rappresentazione lessico-grammaticale dell'ingannare e del mentire}

Se, con Rorty, il mondo non è né vero né falso, mentre lo sono i discorsi sul mondo:

Si deve distinguere tra l'affermazione che il mondo è là fuori e l'affermazione che la verità è là fuori. Dire che il mondo è là fuori, che non è una nostra creazione, equivale a dire, con il senso comune, che la gran parte di ciò che è nello spazio e nel tempo è l'effetto di cause che prescindono dagli stati mentali dell'uomo. Dire che la verità è là fuori equivale a dire, semplicemente,

43. J. Habermas, Theorie des Kommunikativen Handelns Bd. I Handlungsrationalität und geselleschaftliche Rationalisierung, Frankfurt am Main: Surhkamp, 1981 (trad. it. Teoria dell'agire comunicativo vol. I Razionalità nell'azione e razionalizzazione sociale, Bologna: il Mulino 1997, p. 395-396).

44. Cfr. I. KanT, Die Metaphysik der Sitten, 1797 (trad. it. a cura di G. Vidari, La metafisica dei costumi, Bari: Laterza, 2001).

45. Cfr. P. Grice, "Logic and Conversation», in P. Cole, J. L. Morgan (a cura di), Syntax and Semantics - Speech Acts, New York-London: Academic Press, 1975, p. $41-58$ (trad. it. "Logica e Conversazione» in M. SBISÀ (a cura di), Gli atti Linguistici. Aspetti e problemi di filosofia del linguaggio, Milano: Feltrinelli, 1987, p. 199-219, ripubblicato in P. CASALEGNO et alii, Filosofia del Linguaggio, Milano: R. Cortina, p. 221-244. 
che dove non ci sono enunciati non c'è verità, che gli enunciati sono componenti dei linguaggi umani, e che i linguaggi umani sono creazioni umane. La verità non può essere là fuori — non può esistere indipendentemente dalla mente umana - perché non lo possono gli enunciati, Il mondo è là fuori, ma le descrizioni del mondo non lo sono. Solo le descrizioni possono essere vere o false. Il mondo di per sé - a prescindere dalle attività descrittive degli uomini. ${ }^{46}$

gli enunciati e i discorsi menzogneri non possono, però, essere individuati perché, come già sapeva il dio della critica e dello scherno Momo, il cuore umano non è alla vista e, quindi, l'eventuale cor duplex di coloro che li pronunciano. Per tale ragione, non possiamo costruire una grammatica della menzogna, ma una grammatica sull'inganno e sulla menzogna. È possibile, cioè, individuare il lessico e la grammatica degli enunciati che parlano della menzogna, di ciò che è disponibile all'uso dei parlanti quanto si imbattono nei discorsi su di essa.

La forma della rappresentazione è quella definita in modo standard nella letteratura lessico-grammaticale a partire da Gross (1975) e, per l'italiano, dai già citati studi di Elia, Martinelli (1981) e D'Agostino e di D’Agostino (1993). Dato il sotto-insieme delle forme lessicali L e il sotto-insieme delle proprietà di frase $\mathrm{P}$, tale rappresentazione assume la forma di una tassonomia del tipo:

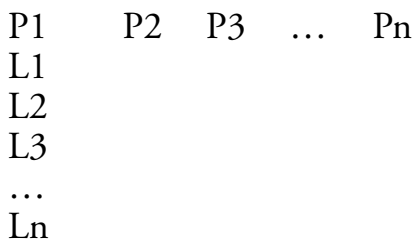

e, testando l'accettabilità di ogni elemento $\mathrm{P}$ per ogni elemento $\mathrm{L}$, si otterrà una tassonomia di tipo binario del tipo:

$\begin{array}{llllll}\text { P1 } & \text { P2 } & \text { P3 } & \ldots & \text { Pn } & \\ \text { L1 } & + & - & - & + & + \\ \text { L2 } & + & + & - & - & - \\ \text { L3 } & - & + & + & - & - \\ \text {.. } & - & - & + & - & - \\ \text { Ln } & - & + & - & + & -\end{array}$

Per la natura ed il tipo delle proprietà di forme di frase utilizzate sistematicamente nei lavori lessico-grammaticali sull'italiano si rimanda, in particolare, al saggio citato di D’Agostino. 
In quest'occasione è stato selezionato un numero ridotto di entrate lessicali semplici e composte ${ }^{47}$ concernenti la coppia menzogna-inganno ${ }^{48}$ ed un numero ridotto di proprietà grammaticali che si commenteranno. Per le prime sono presentate venti entrate lessicali e dodici proprietà di frase ottenendo la classificazione presente in (2.3.).

È stata altresì indicata la presenza delle entrate lessicali nel Lessico di frequenza della lingua italiana contemporanea (L.I.F.) e nel Lessico di frequenza dell'italiano parlato (L.I.P.)

\subsection{Le proprietà analizzate}

Va ricordato che, nella classificazioni lessico-grammaticali, è possibile attribuire al termine proprietà $(\mathrm{P})$ la comune accezione intuitiva di "caratteristica» di un elemento lessicale che fa sì che questo appartenga ad un determinato insieme, faccia cioè parte di una classe, cioè di una collezione di elementi considerata come un tutto. Allo stesso tempo, per noi, se ogni P si concretizza nella possibilità, per un certo elemento lessicale, di realizzarsi in una data forma di frase, proprietà e forma di frase sono nozioni associate. Va precisato ancora che, poiché nella definizione di una classe normalmente intervengono più proprietà convergenti, per dire che una certa entrata verbale è membro di una certa classe di costruzioni, essa deve presentare più di una $\mathrm{P}$, quindi più forme di frase, pertinenti per quella classe, devono rivelarsi accettabili in relazione a quella entrata. Nelle classificazioni di tale tipo, nella maggioranza dei casi, ogni classe, come si è detto, è definita dalla contemporanea presenza di più di una $\mathrm{P}$; naturalmente le varie proprietà che convergono nella definizione di una classe saranno tra loro di tipo diverso. In modo standard sono state applicate comunemente:

1) proprietà strutturali concernenti il numero e la posizione degli argomenti dell'operatore, con l'esclusione, ovviamente, dal calcolo dei cosiddetti complementi «non pertinenti» o «circostanziali» e quelli «di nome», fondamentalmente perché entrambi i tipi costituiscono, generalmente, riduzioni di altre frasi e in tal senso, sarà possibile ritenere che le $\mathrm{P}$ strutturali concernenti una determinata entrata saranno quelle che riguardano la realizzazione sintattica della relazione che si stabilisce tra un operatore ed i suoi argomenti, così come essa si manifesta nella frase di base associata definizionalmente a quel determinato verbo o uso verbale. Quelle che qui vengono definite proprietà strutturali prevedono fondamentalmente l'analisi delle varie posizioni complemento e, di riflesso, anche la considerazione distribuzionale delle preposizioni che si legano ad un complemento;

47. Per la distinzione tra forme lessicali semplici e forme lessicali composte si rimanda ancora una volta al saggio citato di D'Agostino (1983).

48. Le forme lessicali dell'italiano d'uso che riguardano tale coppia arrivano a circa 130 entrate. 
2) proprietà distribuzionali concernenti i vincoli di selezione operanti sulle posizioni occupate dai vari argomenti. Con P distribuzionali ci si vuole riferire in modo più specifico a quell'insieme di forme di frase che servono ad identificare i caratteri della selezione distribuzionale delle forme nominali che co-occorrono con la specifica entrata verbale nelle varie posizioni previste e che coincidono con gli argomenti dell'operatore. Caratteri che vedono realizzarsi nelle varie posizioni (soggetto e complementi) membri di classi di $\mathrm{N}$ di tipo e consistenza variabili. Attraverso di esse si concretizza la selezione operata dall'operatore e, in tal senso, le P distribuzionali procedono identificando classi di $\mathrm{N}$ che variamente si collocano lungo quel segmento che va da quello che si può definire «il polo della minore restrizione» a quello che si può chiamare «il polo della maggiore restrizione»;

3) proprietà trasformazionali che costituiscono un insieme, complesso e estremamente differenziato al proprio interno, di forme di frase correlate sistematicamente in termini di rapporti parafrastici a quelle che vengono individuate come frasi di base associate definizionalmente all'entrata verbale e, allo stesso tempo, frutto di manipolazioni di diversa natura e complessità. Il termine "trasformazionale» è impiegato non nell'accezione di tipo derivazionale per la quale il rapporto tra due frasi è orientato da una base alla sua trasformata $(\mathrm{a} \rightarrow \mathrm{b})$, ma piuttosto in un'accezione direttamente collegata all'uso harrisiano della nozione di equivalenza come caso particolare della più generale variazione morfofonemica. ${ }^{49}$

In questa occasione, sono state analizzati tre tipi di proprietà e cioè: proprietà di restrizione di selezione, proprietà strutturali, una proprietà trasformazio-

49. Cfr. D’Agostino, op. cit., p. 197-198. A tale riguardo, ci si richiamava direttamente all'affermazione harrisiana per la quale: «En partant de l'ensemble des discours, c'est-à-dire de tout ce qui est dans la langue, nous découvrons qu'il existe une certaine relation d'équivalence qui determine une partition des discours; les discours qui figurent dans une même classe d'équivalence seront appelés des transformées paraphrastiques (les unes des autres). Pratiquement, la relation est constituée par le fait qu'un opérateur (ou un argument) peut prendre une parmi quelques variantes de forme phonémique (plus rarement des variantes de position) lorsqu'il est cancaténé avec son argument ou son opérateur. Les variantes sont essentiellement des formes zéro, des pro-mots (e.g. pronoms) et des attachements (e.g. des suffixes)...Partant des discours de stricte concaténation, nous voyons que lorsqu'un opérateur se concatène à ses arguments, ses arguments ou lui même peuvent prendre des variantes de formes (ou de position). Ces variantes sont en génèral facultatives, de sorte que les discours de stricte concaténation ne sont pas remplacés par celle-ci. Cependant, dans des nombreuses langues, certaines des variantes sont obligatoires lors de certaines concaténations, auquel cas le discours de concaténation non modifié n'exixte pas, mais est remplacé par la forme modifié...Le changement de forme n'ajoute aucune information objective à ces discours. Il ne modifie non plus aucune des relations créés par l'opération de concaténation, celles-ci sont uniquement les relations ordonnées de contraintes entre opérateurs et arguments, et elles incluent l'introduction partiellement ordonné des opérateurs dans le discours» (Z. S. HARRIS, Notes du cours de syntaxe, Paris: Ed. du Seuil, 1976, p. 37-39). 
nale ed una proprietà di correlazione morfo-fonemica. Per le prime si è testata l'accettabilità dell'equivalenza distribuzionale tra sintagmi nominali e frasi, ad esempio:

\section{$N_{0} V N_{1} u m$ obbl}

Che $F V N_{1}$ um obbl

che si contrappongono in base alla possibilità di registare in posizione soggetto unicamente un sintagma nominale o una frase:

( Che tu sia partito per Roma + Max) ha buggerato Maria

(Che tu sia partito per Roma + Max) ha ingannato Maria

Per le seconde si è testata la possibilità di registrare la presenza di un sintagma nominale o di un sintagma preposizionale in posizione oggetto:

Max ha beffato Maria

Max si è beffato di Maria

Come proprietà di correlazione morfonemica si è testata la possibilità di una forma nominale associata $(V-n)$, rintracciandola per tutte le forme lessicali semplici e per una composta:

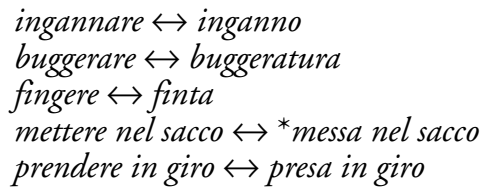

e collegando tale proprietà alla proprietà trasformazionale di nominalizzazione con i due Verbi supporto fare e dare:

Max si è beffato di Maria $\leftrightarrow$ Max si è fatto beffe di Maria

Max ha fregato Maria $\leftrightarrow$ Ma ha dato una fregatura a Maria

\subsection{La tavola}

Qui di seguito si presenta la tavola relativa agli esempi selezionati. 


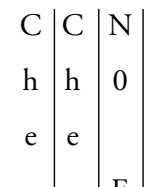

E

F

S

\begin{tabular}{l|l|l} 
V & E & E
\end{tabular}

S $\quad$ R

\begin{tabular}{l|l|l}
$\mathrm{N}$ & $\mathrm{S}$ & $\mathrm{E}$
\end{tabular}

$1 \mathrm{e}$

$\begin{array}{lll}\mathrm{u} & \mathrm{r} & \mathrm{D}\end{array}$

m e e

o $t$

b $\mathrm{D}$

b e $\mathrm{V}$

$\mathrm{l} \mathrm{t} \mathrm{n}$

V

n

$-++$

$--$

$+++$

$--$

++- BUbBOLARE

++- BUGGERARE

-++ CONTRAFFARE

- - - DARE A VEDERE

- - - DARE A INTENDERE

- - - DEFORMARE

- + + DISSIMULARE

- - - DISTORCERE

$-++\mid$ FALSIFICARE

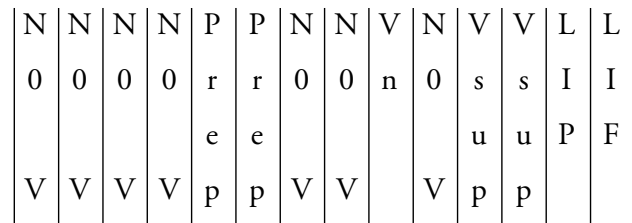

$\begin{array}{lllllllllllllll}\text { N } & \text { N } & \text { N } & \text { P } & \text { d } & \text { a } & \text { C } & \text { d } & & \text { u } & \text { F } & \text { D }\end{array}$

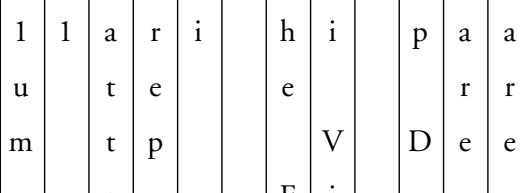

\begin{tabular}{l|l|l|l|l|l|l|l|l} 
o & r & & F & i & e \\
b & i & N & & n & t
\end{tabular}

$\mathrm{b}$

b 1

1

$\mathrm{u}$

$\mathrm{t}$

O

f

V

W n

W

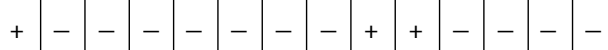

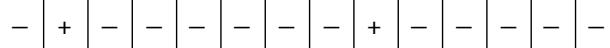

$+-\begin{array}{llllllllllllll}- & - & - & - & - & - & - & + & - & + & - & - & -\end{array}$

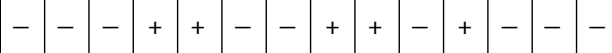

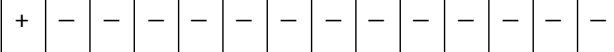

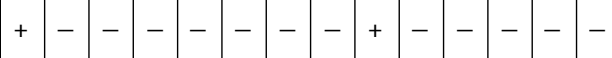

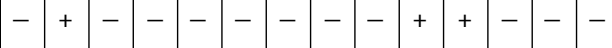

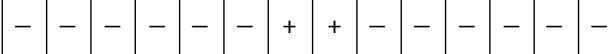

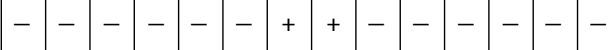

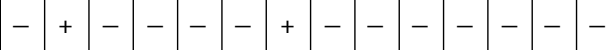

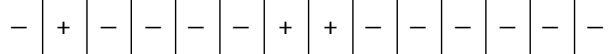

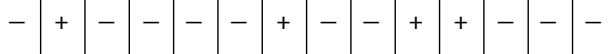

- + + $-|-|-|-|+|-|+|-|+|-|-\mid-$ 


$$
\begin{aligned}
& \text { C }|\mathrm{C}| \mathrm{N} \\
& \text { h h } 0 \\
& \text { e e } \\
& \text { E } \\
& \text { F } \quad \text { F } S \\
& \text { S } \\
& \text { V E } \\
& \text { s } \mathrm{R} \\
& \begin{array}{l|l|l}
\mathrm{N} & \mathrm{s} & \mathrm{E}
\end{array} \\
& 1 \text { e D } \\
& \begin{array}{lll} 
& \mathrm{r} & \mathrm{e}
\end{array} \\
& \mathrm{m} \text { e } \mathrm{t}
\end{aligned}
$$$$
\text { o }
$$$$
\text { b } \quad \text { D } \mathrm{V}
$$$$
\text { b } \quad \text { e } \mathrm{N}
$$$$
\mathrm{l} \mathrm{t}
$$$$
\text { V }
$$$$
\text { n }
$$

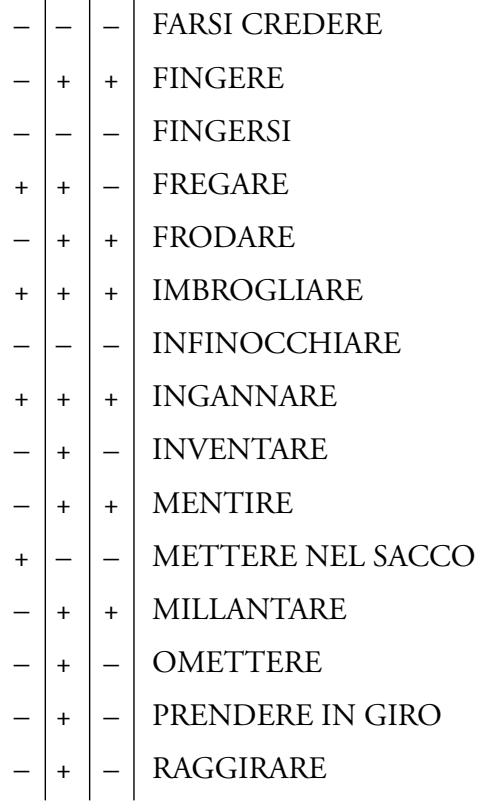

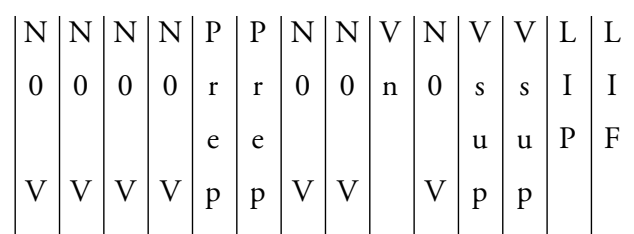

\begin{tabular}{l|l|l|l|l|l|l|l|l|l|l|l|l}
$\mathrm{N}$ & $\mathrm{N}$ & $\mathrm{N}$ & $\mathrm{P}$ & $\mathrm{d}$ & $\mathrm{a}$ & $\mathrm{C}$ & $\mathrm{d}$ & & $\mathrm{u}$ & $\mathrm{F}$ & $\mathrm{D}$
\end{tabular}

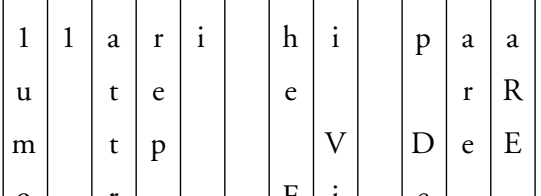
o $\quad$ r $\quad$ F i $\quad$ e

b $\quad$ i $\quad \mathrm{N}$

$\begin{array}{llll}\text { b } & \text { b } & 1\end{array}$ 1 $\mathrm{u}$ $\mathrm{t}$ V W

n o

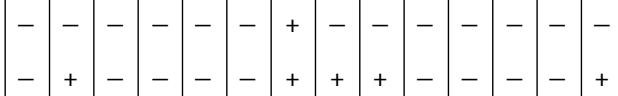

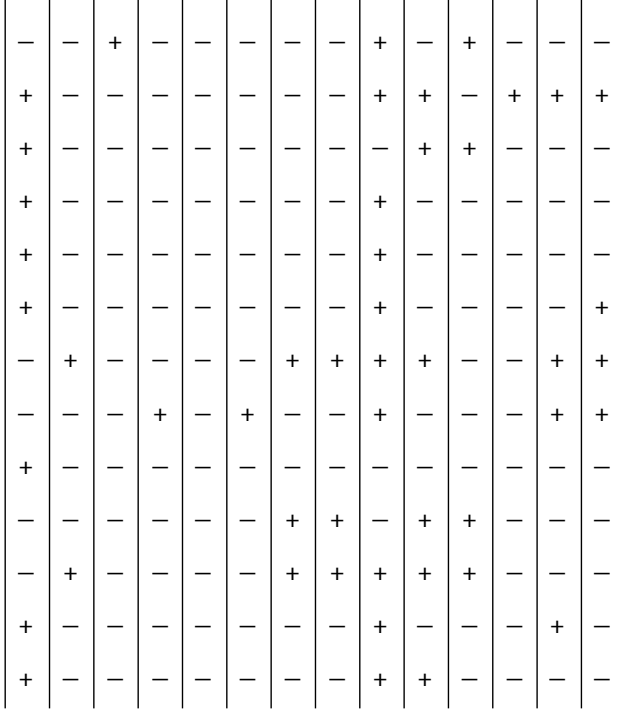


Dalla lettura della tavola si ricavano differenti informazioni concernenti in primo luogo lo statuto di operatore elementare o non elementare degli operatori e l'appartenenza di questi a classi diverse. Infatti si rileva la seguente situazione:

(1) Operatori elementari Onn

abbindolare artefare contraffare deformare distorcere falsificare frodare imbrogliare infinocchiare mentire mettere nel sacco prendere in giro raggirare

tale tipologia di operatori si realizza in frasi che presentano una struttura come le seguenti:

$\mathrm{N}_{0} \mathrm{~V} \mathrm{~N}_{1}$ um obbl

Max ha imbrogliato Eva

$\mathrm{N}_{0} \mathrm{~V} \mathrm{~N}_{1}$

Max ha deformato il racconto di Eva

$\mathrm{N}_{0} \mathrm{~V}$ Prep $\mathrm{N}_{1}$

Max ha mentito a Maria

(2) Operatori non elementari Ono

beffarsi dare a vedere dare a intendere dissimulare fingere inventare millantare omettere

$\mathrm{N}_{0} \mathrm{~V}$ Che $\mathrm{F}$

Max ha finto che Maria era partita

cui si associa l'eventuale riduzione all'infinitiva con co-referenza del soggetto:

$\mathrm{N}_{0} \mathrm{~V}$ di $\operatorname{Vinf}^{0} \mathrm{~W}$

Max ha finto di partire per la Tunisia

(3) operatori non elementari Oon

beffare bubbolare buggerare fregare ingannare

Che F V N

Che Max fosse partito per la Tunisia ha ingannato Maria

Sempre a proposito dei soggetti di tipo frastico va notato che si registrano anche costruzioni a supporto del tipo:

Che F Essere Det V-n

Che Max sia partito per la Tunisia è stata una fregatura 
Va notato inoltre che nel caso dei verbi farsi credere e fingersi le forme di frase con in posizione $\mathrm{N}_{1}$ un attributo del soggetto:

Max (si fa credere + si finge) magistrato

siano riconducibili a una struttura di tipo causativo:

Max fa credere che è magistrato

* Max fa credersi magistrato

Max si fa credere magistrato

Oppure di tipo completivo come;

Max finge che è magistrato

Max finge di essere magistrato

Max si finge magistrato

Va registrata la possibilità di $V$ sup estensioni di essere come rivelarsi, dimostrarsi, costituire, rappresentare. Infine appare significativa la quasi totale assenza delle forme verbali in questione dai lessici di frequenza esaminati e la contestuale registrazione in questi ultimi delle corrispondenti forme nominali associate come imbrogliare $\leftrightarrow$ imbroglione.

\section{Conclusioni}

In questo contributo si è tentato di mostrare come sia possibile convertire in senso harrisiano le nozioni di «apparato significante» di origine greimasiana e di «vocabolario» in senso rortriano in un'analisi linguistica fondata sulla grammatica harrisiana in operatori e argomenti e sulla procedura di rappresentazione lessico-grammaticale elaborata da Maurice Gross. Un'indagine analoga è stata effettuata su una parte del vocabolario delle passioni, in particolare dell'ira e dell'odio, e verrà presentata in una prossima occasione. 